Article

\title{
Development and Preliminary Trajectory Verification of the Electromotor-Driven Parallel External Fixator for Deformity Correction
}

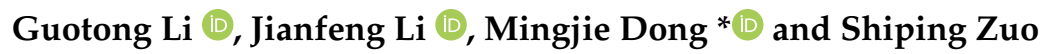 \\ Beijing Key Laboratory of Advanced Manufacturing Technology, Faculty of Materials and Manufacturing, \\ Beijing University of Technology, Beijing 100124, China; B201801033@emails.bjut.edu.cn (G.L.); \\ lijianfeng@bjut.edu.cn (J.L.); zuosp@emails.bjut.edu.cn (S.Z.) \\ * Correspondence: dongmj@bjut.edu.cn
}

Received: 9 November 2020; Accepted: 16 December 2020; Published: 18 December 2020

Featured Application: The developed electromotor-driven parallel external fixator can be automatically adjusted according to the adjustment strategy to reduce the involvement of the patient and can also be used as a teaching tool of medical training for clinicians to learn deformity correction techniques.

\begin{abstract}
External fixators are widely used in deformity correction based on distraction osteogenesis. Traditionally, the rods are manually operated by patients several times a day, which will ensure the patient's compliance, accumulative adjustment error, and trajectory deviation. To reduce the patients' compliance and the complexity of adjustment, an electromotor-driven parallel external fixator is developed to gradually correct the deformity, which allows the fixator to be automatically adjusted and can correct any three-dimensional deformity with continuous stability. Two adjustment strategies are proposed through different trajectory control methods based on the inverse kinematics solution, and the trajectory and bone shape are generated to investigate the characteristics of the new bone more intuitively. The range of motion is performed utilizing the numerical searching method to assess the fixator's correction capability. Finally, the trajectory verification experiment is carried out using the artificial bone model to perform the two adjustment strategies. The results show that the developed external fixator has high correction accuracy with $0.0172 \mathrm{~mm}$, and can accurately and safely realize the preset correction trajectory. The developed fixator system can also be used as a teaching tool for medical training for clinicians to learn deformity correction technology.
\end{abstract}

Keywords: external fixator; deformity correction; adjustment strategy; correction capability; trajectory verification

\section{Introduction}

The external fixator is a well-established technique in distraction osteogenesis which stimulates the body's self-healing capacity to gradually form new tissue [1,2]. The classical Ilizarov ring fixator has obtained good clinical results in correcting tibial deformities [3]. However, the configuration of the Ilizarov frame needs to be modified constantly, which is a very complex process, especially in the implementation of multiplane correction [4]. Taylor spatial frame (TSF) allows multiplane correction without modifying the configuration of the frame to achieve continuous stability [5]. Traditionally, clinicians complete the deformity correction by manually adjusting the rods of the external fixator according to the prescription and the number of adjustments is limited by the patient's compliance to about four daily steps [6]. Complicated manual adjustment leads to accumulated adjustment error and 
trajectory deviation. Adjusting the rod length of TSF in sequence will cause the trajectory of the bone end to reach five intermediate configurations before fully adjusting all the rods, which will make the actual trajectory deviate from the ideal correction trajectory [7].

In general, the patient cannot check the current correction status and the remaining deformity in real-time and does not know whether the correction plan has been implemented on that day, which will lead to an unstable emotion and cause the patient to forget to implement the plan [8]. The trajectory deviation caused by the accumulated adjustment errors is also an issue in practical application. Once the trajectory deviation occurs, it is necessary to take an X-ray to reestablish the correction strategy, which will increase the patient's radiation exposure [9]. Du et al., developed a teleoperation robot system based on a removable series-parallel mechanism that can be used for acute correction of long bone fractures $[10,11]$. However, deformity correction is based on the distraction osteogenesis to stimulate the body's self-healing capacities to gradually form new tissue, which requires the patient to wear the external fixator for a long time [1]. By mounting six rods on Ilizarov rings, K Seide et al., developed the hexapod system which can plan and correct deformities with computer assistance [12]. Several researchers have described the advantages and precision of similar mechanisms [13-15]. However, they cannot reflect the deflection of the actual and the target trajectory during the correction, and there is no trajectory verification of the pre-operative correction trajectory. Meanwhile, the effect of the orientation of the distal bone end on bone trajectory has not been considered during the implementation of different correction strategies. Additionally, kinematics and mathematical derivations of the bone end trajectory and how to model the resulting bone shape for each correction strategy have rarely been studied under an external fixator.

The range of motion ( $\mathrm{ROM})$ is a crucial indicator to assess the correction capability of the external fixator, which must have a sufficient ROM to accommodate the correction requirement of different indications; otherwise, the correction plan will not be carried out [16]. At present, clinicians evaluate the ROM of the external fixator only by investigating the individual displacement and angle capabilities [11,12], instead of investigating the ROM from a three-dimensional perspective. A numerical searching method is provided to investigate the ROM to assist clinicians in more intuitively assessing the fixator's correction capability.

An electromotor-driven parallel external fixator is developed for tibia deformity correction based on the Stewart platform. It allows automatic adjustment and gradual correction of the deformity, reducing the involvement of the patient. The preliminary trajectory verification experiments are carried out utilizing the artificial bone model to investigate the accuracy of the developed fixator system.

\section{Materials and Methods}

\subsection{The Bone-Fixator System}

The developed parallel external fixator is a kind of hexapod fixator based on the Steward platform, which contains two rings, six telescopic rods, six Hooke joints, and spherical hinges. The proximal and distal rings are connected through six telescopic rods, as shown in Figure 1a. The proximal and distal bone segments are vertically and rigidly fixed on the proximal ring and distal ring, respectively, and the axes of the bone segments coincide with the center of the ring. The position and orientation of the distal bone segment can be changed spatially by regulating the length of each rod, and the frame can realize the correction of any three-dimensional deformity without modifying the configuration $[5,17]$. The proximal and distal bone segments are aligned along the anatomical axis of the tibia, which indicates that the correction has been completed and the fixator attains the neutral position. 
(a)

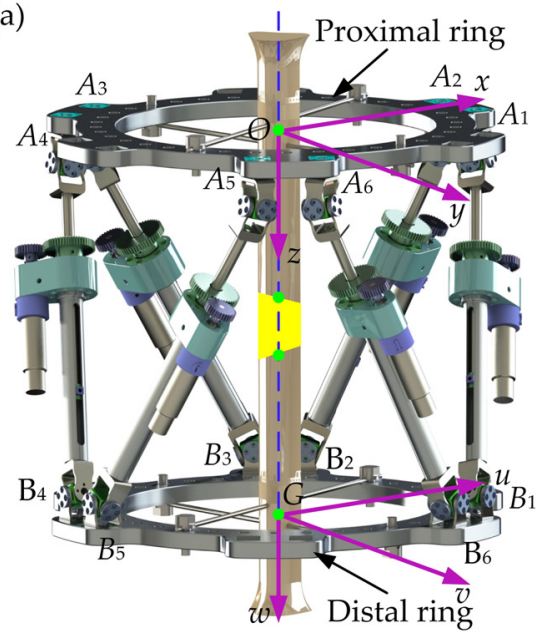

(b)

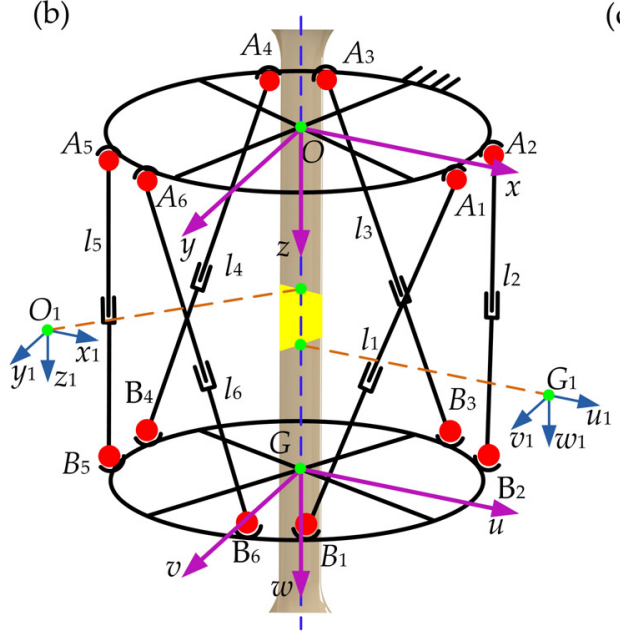

(c)

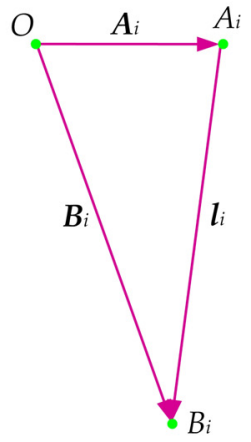

Figure 1. (a) The neutral position of the bone-fixator system. (b) Schematic diagram of the frame. The points $A_{i}(i=1,2, \ldots, 6)$ and $B_{i}(i=1,2, \ldots, 6)$ are the centers of the Hooke and spherical joints.

(c) The closed vector chain used to solve the vector $l_{i}$.

The schematic diagram of the bone-fixator system has been developed, as shown in Figure 1b. The fixed reference frame $O-x y z$ is established at the mass center of the proximal ring with the $x$-axis passing through the midpoint of $A_{1} A_{2}$ and the $z$-axis normal to the ring. The frame $O_{1}-x_{1} y_{1} z_{1}$ is established at the center of the proximal bone segment end, which is parallel to the frame O-xyz. The development of the moving coordinate systems $G-u v w$ and $G_{1}-u_{1} v_{1} w_{1}$ are similar to those of frames $O-x y z$ and $O_{1}-x_{1} y_{1} z_{1}$. The orientation of the frame $O-x y z$ and the frame $G-u v w$ is coincident in the neutral position.

\subsection{Inverse Kinematic Solution}

Presuming that $\theta_{1}, \theta_{2}$, and $\theta_{3}$ are the rotating angles of the frame $G-u v w$ concerning the frame $O-x y z$ in the sequence of $x-, y$-, and $z$-axes, the posture of $G$ can be achieved through the following coordinate transformation:

$$
T_{G}^{O}=T_{G_{1}}^{O} T_{G}^{G_{1}}=\left[\begin{array}{cc}
R_{G}^{O} & P_{G}^{O} \\
0 & 1
\end{array}\right]=\left[\begin{array}{cc}
R_{G_{1}}^{O} & P_{G_{1}}^{O} \\
0 & 1
\end{array}\right]\left[\begin{array}{cc}
R_{G}^{G_{1}} & P_{G}^{G_{1}} \\
0 & 1
\end{array}\right]
$$

where

$$
\left\{\begin{array}{c}
R_{G_{1}}^{O}=R_{G^{\prime}}^{O} \quad P_{G_{1}}^{O}=\left[\begin{array}{lll}
x_{G_{1}} & y_{G_{1}} & z_{G_{1}}
\end{array}\right]^{\mathrm{T}} \\
R_{G}^{G_{1}}=\left[\begin{array}{lll}
1 & 0 & 0 \\
0 & 1 & 0 \\
0 & 0 & 1
\end{array}\right], \quad P_{G}^{G_{1}}=\left[\begin{array}{lll}
0 & 0 & h_{L}^{\prime}
\end{array}\right]^{\mathrm{T}}
\end{array}\right.
$$

The $R_{G}^{O}$ is the homogeneous transformation matrix; the $h_{L}{ }_{L}$ is the length of the distal bone segment $G G_{1} ; P_{G_{1}}^{O}$ is the displacement vector of $G_{1}$ concerning the frame $O-x y z$, and $P_{G}^{G_{1}}$ is the displacement vector of $G$ to the frame $G_{1}-u_{1} v_{1} w_{1}$.

The inverse kinematics of the fixator can be resolved through the closed-loop method. The closed vector chain is shown in Figure 1c. The vector $l_{i}(i=1,2, \ldots, 6)$ of rod length can be achieved by Equation (2).

$$
\boldsymbol{l}_{i}=\boldsymbol{B}_{i}-\boldsymbol{A}_{i}=\left[\begin{array}{lll}
l_{i x} & l_{i y} & l_{i z}
\end{array}\right]^{T}
$$


where $A_{i}, B_{i}(i=1,2, \ldots, 6)$ are position vectors concerning origin $O$ in the frame $O-x y z$. Then, the rod length $\boldsymbol{l}_{i}(i=1,2, \ldots, 6)$ is achieved through Equation (3).

$$
\left|\boldsymbol{l}_{i}\right|=\sqrt{l_{i x}^{2}+l_{i y}^{2}+l_{i z}^{2}}
$$

Thus, the correction prescription, the length of the rods $l_{i}(i=1,2, \ldots, 6)$ at each step, can be achieved through the inverse kinematic solution.

\subsection{The Algorithm for Trajectory Planning}

\subsubsection{Equal Joint Adjustment}

Clinically, the deformed bone is cut into two segments at the center of rotation of angulation (CORA) and stabilized with a fixator [18]; then, gradual correction can be achieved based on distraction osteogenesis (DO). It is presumed that the total correction step is $n$ steps, and the length $l_{1}\left(l_{11}, l_{21}, l_{31}\right.$, $\left.l_{41}, l_{51}, l_{61}\right)$ and $l_{n}\left(l_{1 n}, l_{2 n}, l_{3 n}, l_{4 n}, l_{5 n}, l_{6 n}\right)$ represent the length of the rods at the initial and target position, respectively. The clinician usually splits the length difference of a rod from the initial deformed position to the target position into equal parts and regulates the rod length according to the adjustment prescription to accomplish the deformity correction. The value of the length difference of each rod is expressed as Equation (4):

$$
\Delta l_{i}=l_{\text {in }}-l_{i 1}
$$

where $l_{i 1}$ and $l_{\text {in }}(i=1,2, \ldots, 6)$ represent the initial length and the final length of the $i$ th rod, respectively. Then, the value of the length difference of each rod is split into $n$ parts equally, which is described as Equation (5):

$$
\Delta s_{i}=\frac{\Delta l_{i}}{n}
$$

The new length $l_{i j}(i=1,2, \ldots, 6 ; j=1,2, \ldots, n)$ of the rod at step $j$ can be achieved as Equation (6):

$$
l_{i j}=l_{i j-1}+\Delta s_{i}
$$

\subsubsection{Joint Adjustment for Equal Bone Distraction}

It is presumed that $\Gamma_{1}\left(X_{G 11}, Y_{G 11}, Z_{G 11}, \theta_{11}, \theta_{21}, \theta_{31}\right)$ and $\Gamma_{n}\left(0,0, Z_{G 1 n}, 0,0,0\right)$ are the position and orientation of distal bone segment in the initial deformed position and the final desired position, respectively. When the clinician is performing an osteotomy for distraction osteogenesis, the desired trajectory is considered to be a uniformly spaced $(1 \mathrm{~mm})$ linear trajectory, and the desired bone shape is considered as close as possible to be a straight bone [6]. Thus, a strategy that involves the orientation of the bone segment and the shortest growth path, through the method of Cartesian coordinate path control, is proposed to operate the position and orientation to generate a uniformly spaced linear trajectory.

To equally plan the position of the distal bone end, the difference of the position is equally split into $n$ parts, which is indicated as Equation (7):

$$
\Delta x_{G_{1}}=\frac{x_{G_{1 n}}-x_{G_{11}}}{n}, \quad \Delta y_{G_{1}}=\frac{y_{G_{1 n}}-y_{G_{11}}}{n}, \quad \Delta z_{G_{1}}=\frac{z_{G_{1 n}}-z_{G_{11}}}{n}
$$

where $G_{11}\left(x_{G 11}, y_{G 11}, z_{G 11}\right)$ and $G_{1 n}\left(x_{G 1 n}, y_{G 1 n}, z_{G 1 n}\right)$ are position vectors of $G_{1}$ at step 1 and step $n$ in $O-x y z$, respectively. Then, $G_{1 h}\left(x_{G 1 h}, y_{G 1 h}, z_{G 1 h}\right)(h=1,2, \ldots, n)$ is the coordinate of $G_{1}$ at step $h$, which is indicated as Equation (8):

$$
G_{1 h}=\left(\begin{array}{lll}
x_{G_{11}}+h \Delta x_{G_{1}}, \quad y_{G_{11}}+h \Delta y_{G_{1}}, & z_{G_{11}}+h \Delta z_{G_{1}}
\end{array}\right)
$$


To equally plan the orientation of the distal bone, the difference of the orientation is equally split into $n$ parts, which is indicated as Equation (9):

$$
\Delta \theta_{1}=\frac{\theta_{1 n}-\theta_{11}}{n}, \quad \Delta \theta_{2}=\frac{\theta_{2 n}-\theta_{21}}{n}, \quad \Delta \theta_{3}=\frac{\theta_{3 n}-\theta_{31}}{n}
$$

Then, the value of orientation $\theta_{h}\left(\theta_{1 h}, \theta_{2 h}, \theta_{3 h}\right)(h=1,2, \ldots, n)$ at step $h$ is indicated as Equation (10):

$$
\theta_{1 h}=\theta_{11}+h \Delta \theta_{1}, \quad \theta_{2 h}=\theta_{21}+h \Delta \theta_{2} \quad \theta_{3 h}=\theta_{31}+h \Delta \theta_{3}
$$

where $\theta_{1 h}, \theta_{2 h}, \theta_{3 h}(h=1,2, \ldots, n)$ are the angulation deformity of the bone around three directions at step $h$, respectively.

\subsection{The Electromotor-Driven Parallel External Fixator System}

The electromotor-driven parallel external fixator system consists of three parts: the mechanism structure based on the Stewart platform, the control system used to drive the mechanism, and the auxiliary software allowing the clinician to visualize the entire treatment process. The overview of the system configuration is shown in Figure 2. In practical applications, when clinicians use auxiliary software to evaluate preoperative correction strategies, patients do not need to wear the external fixator, which is an independent preoperative operation. The correction strategy is selected based on the results of the preoperative evaluation to guide the orthopedic surgery to ensure good clinical results. When the correction strategy is determined, the corresponding correction trajectory will be downloaded to the microcontroller and stored in the system to execute the trajectory during the correction. After the clinician performs the osteotomy, the external fixator is used for fixation. The patient only needs to perform daily correction according to the corresponding operation module on the control box (the operation can be recorded), which is different from manual adjustment. During the correction, when the patient needs to view the current correction status, the computer and the control box will be connected and communicate through a specific communication protocol to obtain the current correction status. If the current correction status does not need to be viewed, the computer can be removed. The patient will wear the external fixator until the deformity is completely corrected and the new bone is completely calcified. They do not need to carry the equipment for some activities (such as walking) but only need to conduct some corresponding rehabilitation training. Thus, when the patient wears the system for deformity correction, the necessary electrical connections and equipment will not hinder the use of the function.

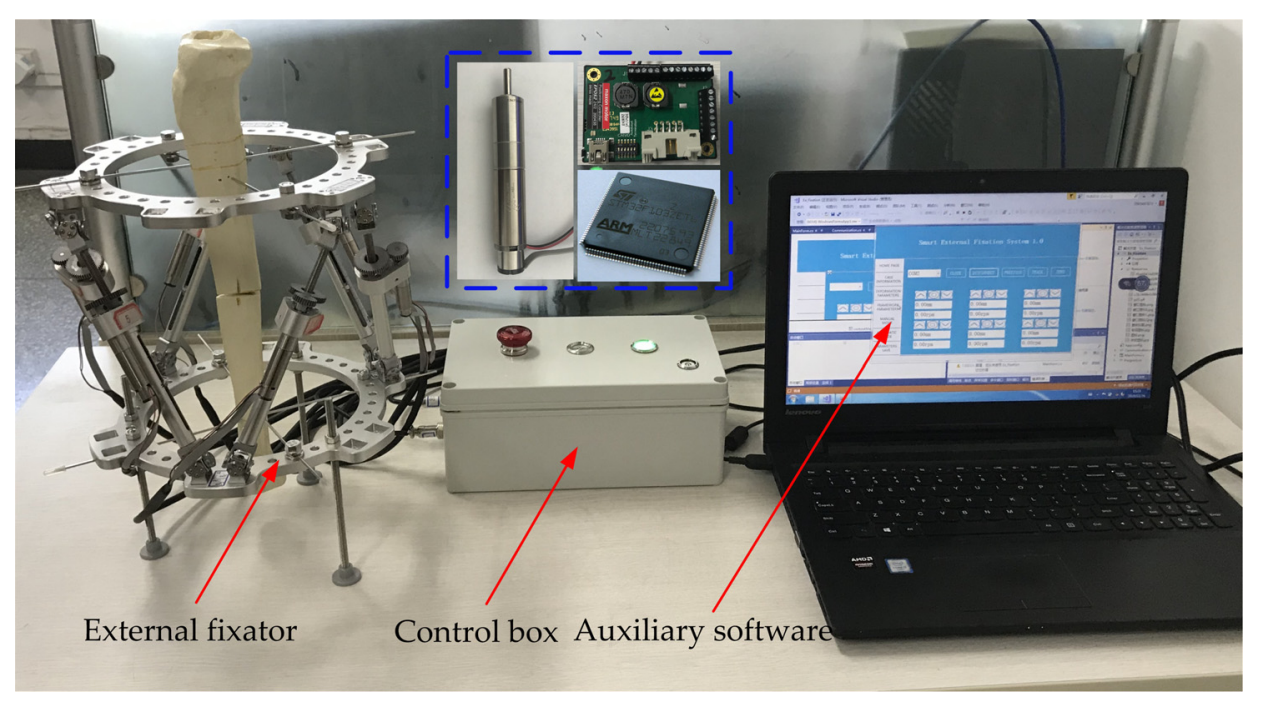

Figure 2. The overview of the system configuration. 


\subsubsection{Structure Design of the Parallel External Fixator}

The developed parallel external fixator is a type of hexapod external fixator, which consists of two rings, six telescopic rods, six Hooke joints, and six spherical hinges. The telescopic rods are connected with the proximal ring and distal ring by the spherical hinges and Hooke joints. The position and orientation of the distal platform can be changed by regulating the rod length and the fixator can correct any three-dimensional malformation without modifying the frame configuration. Compared with the Ilizarov frame, it can be utilized to perform multiplane deformity simultaneous- ly with computer assistance.

The telescopic rod is driven by lead screw transmission, and the DC (Direct Current) servo motor (Maxon DCX 12L) is connected to the lead screw through the gear reduction $\left(i_{1}=5: 3\right)$. There is an incremental encoder at the bottom of the motor (Maxon ENX10 EASY, 1024 CPT), which can feed back the lengths of six rods to the microcontroller in real-time. The motor's weight is $50 \mathrm{~g}$ and the total weight of six motors is $300 \mathrm{~g}$. The motor will be considered to be installed in the outward direction to increase the internal space of the ring, thereby reducing the possibility of interference between the struts of the fixator and the limbs. The additional weights caused by the motor and the corresponding accessories required to install the motor are small, which has a very small impact on the patient's tolerance. Thus, it can still be effectively used clinically with the motor and control unit.

The main parameters of the Maxon DCX 12L are as follows: the maximum rotational speed $n=8810 \mathrm{rpm}$, the rated torque $M=3.88 \mathrm{mN} \cdot \mathrm{m}$, and the reduction ratio of the reduction gearbox (GPX12A) is $i_{2}=62: 1$. The lead screw is adopted with a single thread $\mathrm{T}$ thread ( $p=1 \mathrm{~mm}$ ), which has a good self-locking function. The minimum length between the center of the spherical joint and hook joint is $140 \mathrm{~mm}$ and the maximum length is $210 \mathrm{~mm}$. To increase the flexibility of the structure and avoid joint abrasion during correction, rolling bearings are used at screw nuts, Hooke hinges, and spherical joints. The overall mechanical diagram of the external fixator is shown in Figure 3.

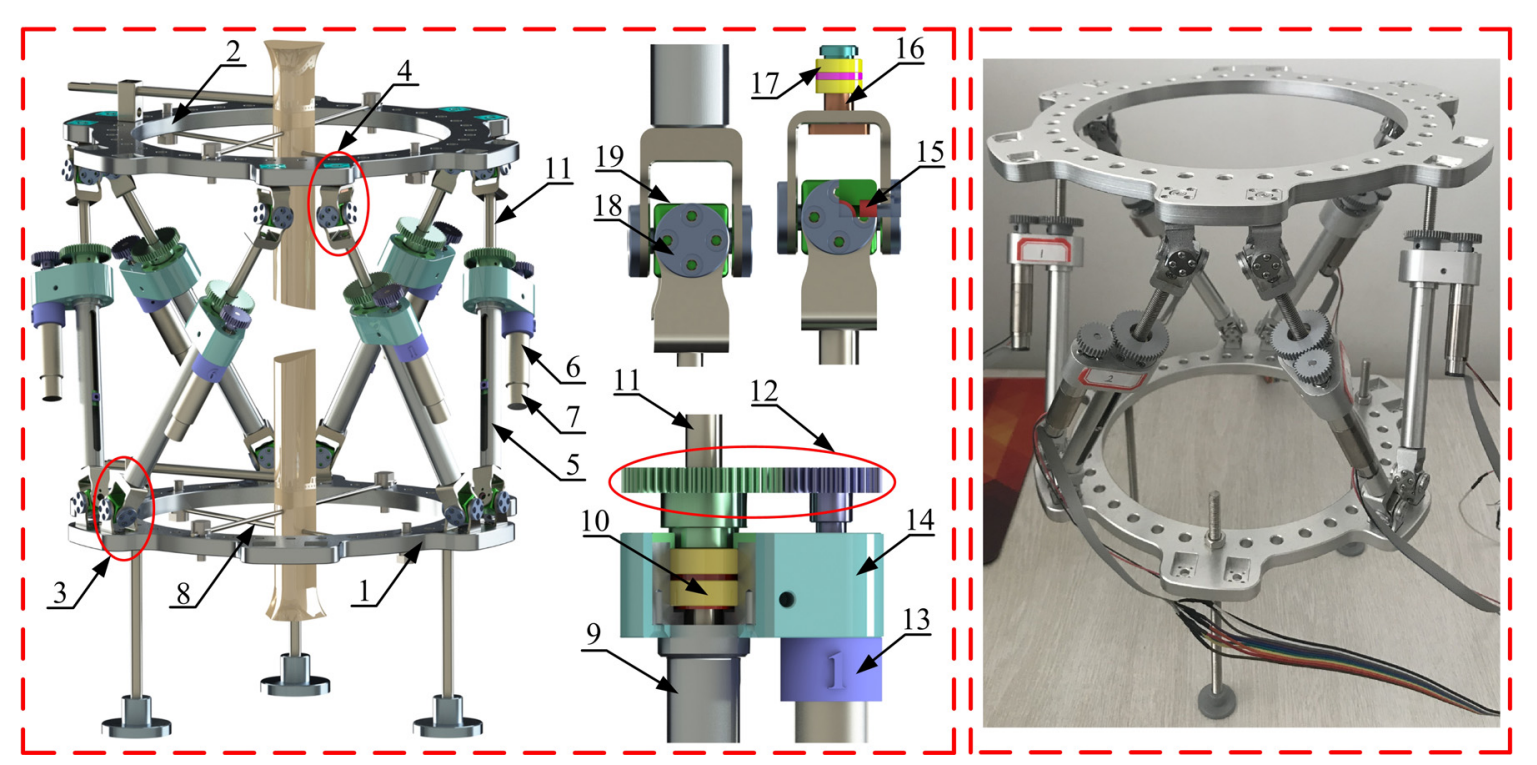

Figure 3. The mechanical diagram of the external fixator. The external fixator consists of (1) proximal ring, (2) distal ring, (3) Hooke joint, (4) spherical hinge, (5) telescopic rod, (6) DC) servo motor, (7) incremental encoder, (8) Kirschner wire, (9) the holder of the telescopic rod, (10) rolling bearing mounted on the screw nut, (11) lead screw, (12) gear reduction, (13) mark number of rod, (14) motor bracket, (15) rolling bearing mounted on the spherical joint, (16) bracket of the spherical joint, (17) bush bearing, (18) end cap, (19) linkage shaft of the Hooke joint.

The value of maximum output force $F_{\max }=2.52 \mathrm{KN}$ and maximum output velocity $V_{\max }=$ $0.89 \mathrm{~mm} / \mathrm{s}$ are acquired. Thus, the motor can provide a larger force/moment to the telescopic rod 
to offset the larger soft tissue force required to pull the soft tissue during the deformity correction. The lower output speed allows for very slow and continuous movement, which is effective for the safety of patients. The maximum output force and velocity can be obtained by Equation (11).

$$
F_{\max }=\frac{2 \pi M \cdot i \cdot \eta}{P}, \quad V_{\max }=\frac{n}{60 i} \cdot P
$$

\subsubsection{The Electromechanical System}

\section{(1) Hardware of the electromechanical system}

The hardware of the electromechanical system mainly includes the motor, incremental encoder, motor driver, and microprocessor, as shown in Figure 4. The power supply voltage of the motor driver (Maxon EPOS2 24/2 390438) is 9 24 V, and the maximum output power is $48 \mathrm{~W}$. The communication interface can be provided in three ways: RS232, CAN (Controller Area Network), and USB 2.0/3.0. In the actual design, the power supply voltage is selected as $12 \mathrm{~V}$, which is less than the human body safety voltage of $36 \mathrm{~V}$. The emergency stop button is set to avoid emergencies, which can ensure the safety of electrical appliances during the use of the system. Furthermore, the CAN bus, mounted with 6 motor drivers (CAN slave station), is used for communication. The STM32 (STM32F103ZET6) chip with the CAN interface is selected as the processor chip. The chip is used as the master station in the CAN network to send control commands to multiple motor drivers (CAN slave stations) when controlling the robot's motion.

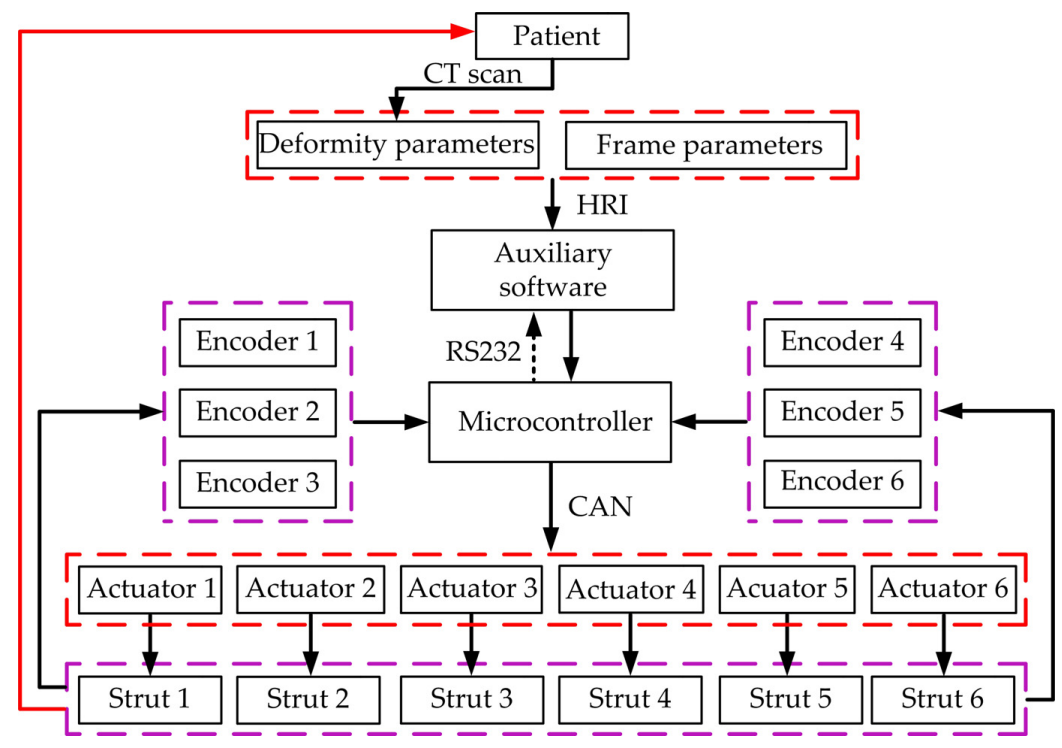

Figure 4. The hardware composition of the electromechanical system.

(2) Mode of the motion control

The incremental encoder is installed at the end of the motor, which can read and feed back the length information of each strut of the external fixator in real-time and obtain the position information of the bone end through the position inverse solution. Then, according to the correction prescription, the microcontroller sends motion instructions to the motor to form a semi-closed loop control mode. Before the correction is implemented, we set the initial position to the zero position. Then, the current correction state can be determined according to the position information feedback from the encoder until the target position is reached.

The external fixator provides three motion control modes: manual adjustment, mechanism reset, and automatic trajectory tracking. Manual adjustment is mainly based on the speed control mode of the CANopen DS402 protocol for motion control of the motor. The automatic trajectory tracking 
is realized based on the position interpolation mode of the motor driver. In the master computer software, the initial position of the mechanism and the increment of each degree of freedom (DOF) are given. The position data are sent to the microcontroller to perform trajectory tracking. The two consecutive trajectory points obtained by the inverse kinematic solution are defined as the reference points $p_{i}$ and $p_{i+1}$ and the velocity $\mathrm{V}$ and time $\mathrm{T}$ are given based on the two reference points. Spline interpolation is used to calculate the functional relationship of the two consecutive points by the motor driver. Then, the position, velocity, and acceleration values are set.

\section{Results}

\subsection{ROM of the External Fixator}

The range of motion ( $\mathrm{ROM}$ ) is a crucial indicator to assess the correction capability of the fixator and can be described as the size of the motion volume, a set of arbitrary reachable postures, of the frame end. The external fixator must have a sufficient ROM to accommodate the correction requirement of different indications; otherwise, the correction plan will not be carried out. The ROM can be described in detail as: giving a searching range in the three-dimensional space, and then detecting whether each posture (corresponding to a set of points) of the frame end is within the searching range. The points that meet the constraints are recorded; otherwise, they will be discarded. Finally, when all the points that meet the conditions are obtained, the reachable area of the frame end can be plotted, which is represented as a closed motion volume.

The diameter of the proximal ring and the distal ring are both $220 \mathrm{~mm}$ and the distance of $A_{i} A_{j}$ $(i j=16,23,45)$ is $32 \mathrm{~mm}$. The constraint condition is the ROM of six telescopic rods; specifically, the minimum and maximum allowable strokes are set to $140 \mathrm{~mm}$ and $210 \mathrm{~mm}$, respectively. With the assistance of MATLAB (R2011a, The Math Works, Inc., Novi, MI, USA), the numerical searching method is adopted to simulate the ROM of the mechanism on basis of the inverse position solution. The simulation results of the position ROM and orientation ROM are shown in Figures 5 and 6.
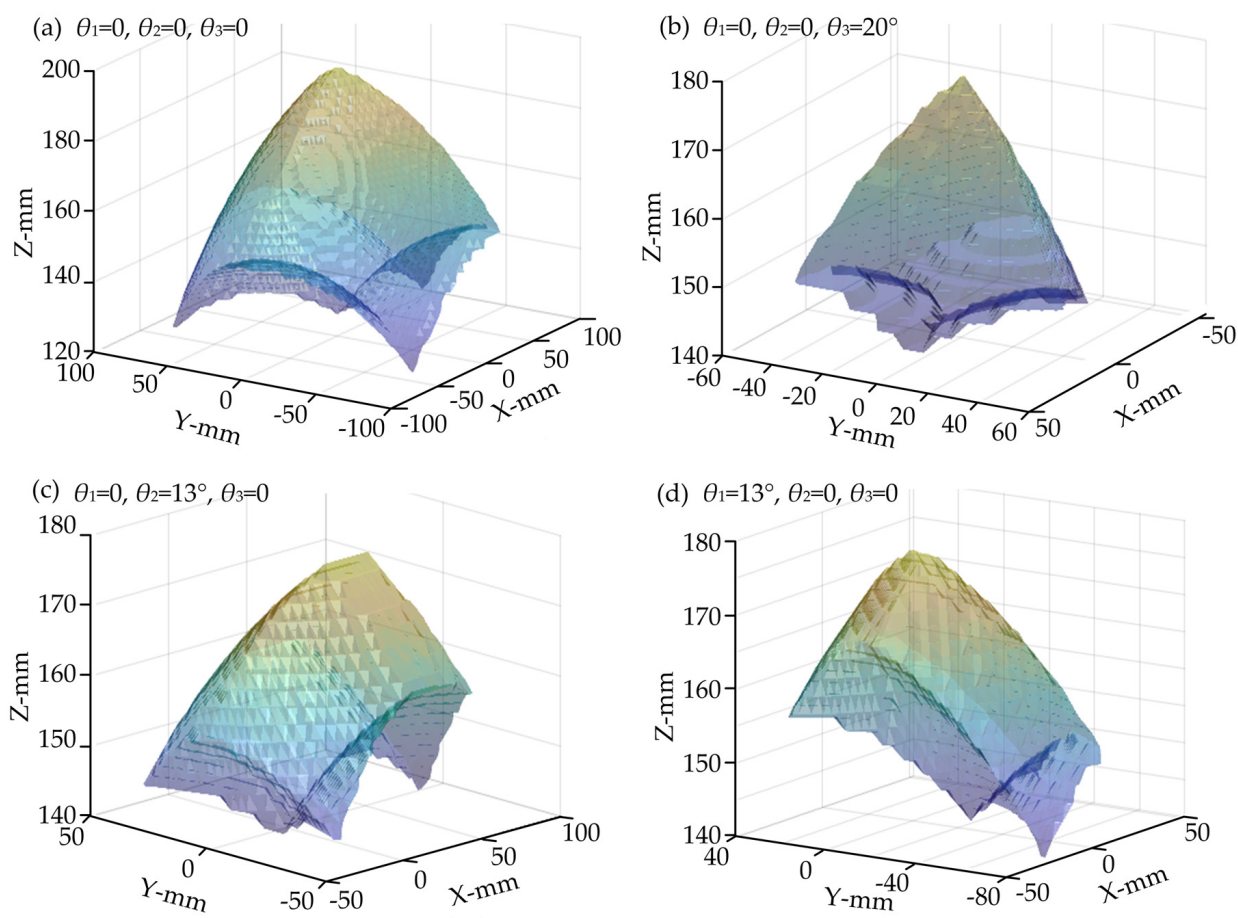

Figure 5. The position range of motion (ROM) of the external fixator. (a) The position ROM of the fixator when the orientation is $(0,0,0)$. (b) The position ROM of the fixator when the orientation is $(0,0,20)$. (c) The position ROM of the fixator when the orientation is $(0,13,0)$. (d) The position ROM of the fixator when the orientation is $(13,0,0)$. 
(a) $X_{G 1}=0, Y_{G 1}=0, Z_{G 1}=159 \mathrm{~mm}$

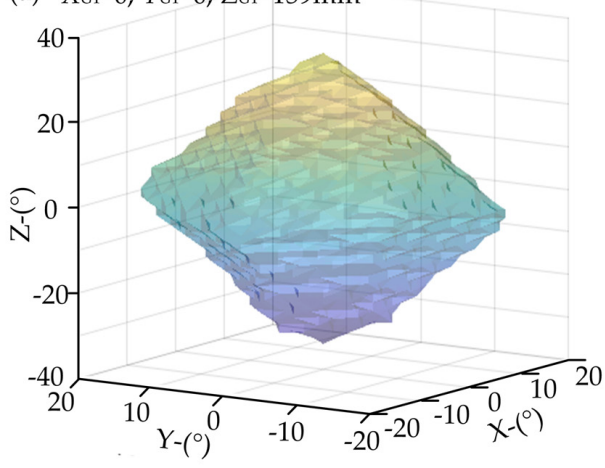

(c) $X_{\mathrm{G} 1}=0, Y_{\mathrm{G} 1}=30 \mathrm{~mm}, Z_{\mathrm{G} 1}=159 \mathrm{~mm}$

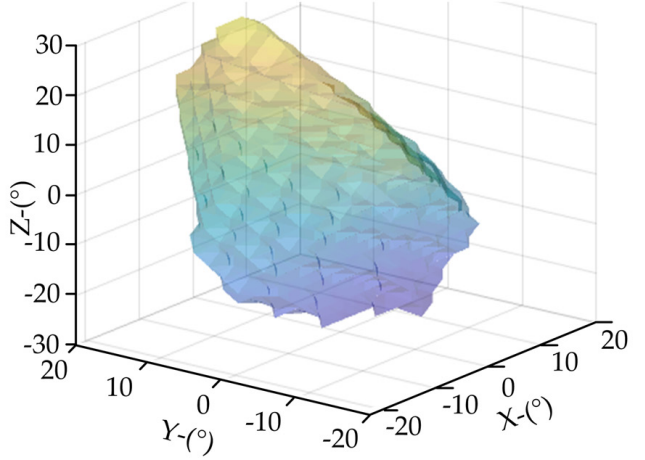

(b) $X_{G 1}=0, Y_{G 1}=0, Z_{G 1}=180 \mathrm{~mm}$

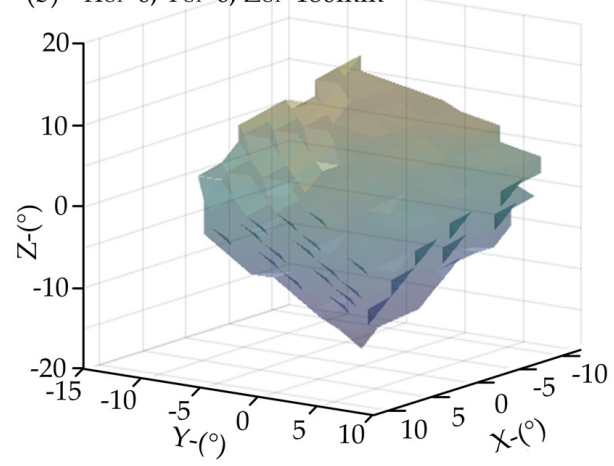

(d) $X_{G 1}=30 \mathrm{~mm}, Y_{G 1}=0, Z_{G 1}=159 \mathrm{~mm}$

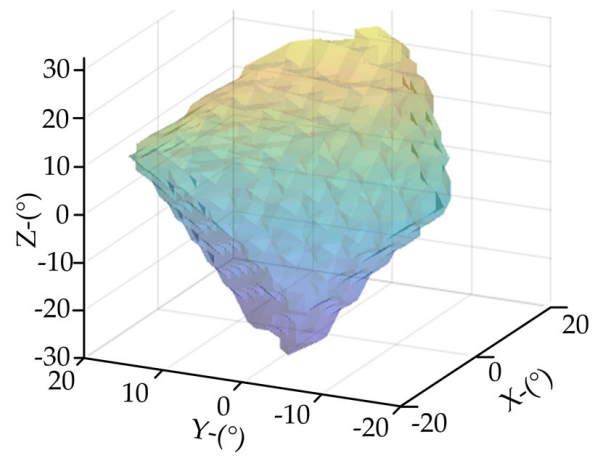

Figure 6. The orientation ROM of the external fixator. (a) The orientation ROM of the fixator when the position is $(0,0,159)$ which is the middle position among the reachable position along the $\mathrm{z}$-axis of the external fixator. (b) The orientation ROM of the fixator when the position is $(0,0,180)$. (c) The orientation ROM of the fixator when the position is $(0,30,159)$. (d) The orientation ROM of the fixator when the position is $(30,0,159)$.

Four different points are set up in this part to view the ROM in the different orientations of the external fixator. The comparison results show that when the orientation is $(0,0,0)$, the fixator has the largest position ROM, as shown in Figure 5a. In this particular configuration, it has a ROM with -92 to $+92 \mathrm{~mm}$ of translation along the $x$-axis, -82 to $+82 \mathrm{~mm}$ of translation along the $y$-axis, and +122 to $+196 \mathrm{~mm}$ of translation along the $z$-axis. It has also been found that the value of $\theta_{2}$ has a greater impact on the displacement along the $y$-axis and the value of $\theta_{1}$ has more constraints on the displacement along the $X$-axis, and the value of $\theta_{3}$ will cause the entire ROM to shrink, as shown in Figure 5b-d.

To investigate the orientation ROM of the external fixator, four different points are set up in different positions. The simulation result shows that when the position is $(0,0,159)$, the middle position among the reachable position along the z-axis of the external fixator, the fixator has the largest position ROM, as shown in Figure 6a. In this particular configuration, it has a ROM with $-18^{\circ}$ to $+18^{\circ}$ of rotation around the $x$-axis, $-20^{\circ}$ to $+20^{\circ}$ of rotation around the y-axis, and $-34^{\circ}$ to $+34^{\circ}$ of rotation around the z-axis. It has also been found that the value of $Y_{G 1}$ has a greater impact on the rotation around the $y$-axis and the value of $X_{G 1}$ has more constraints on the rotation around the $x$-axis, and the value of $Z_{G 1}$ will cause the entire ROM to shrink, as shown in Figure $6 \mathrm{~b}-\mathrm{d}$.

The correction capability of the orientation of the fixator in any position can be determined, represented intuitively in the form of the volume of space-reachable position, and the position operation capability in any orientation of the fixator can also be determined through this method. Meanwhile, this method can also be used to determine whether any given position and orientation are within the correction capability of the external fixator. The maximum position correction capability and the maximum orientation correction capability of the external fixator, obtained through the simulation results, are listed in Table 1. 
Table 1. The maximum correction capability in space-reachable position.

\begin{tabular}{|c|c|c|c|c|}
\hline \multicolumn{4}{|c|}{ The Maximum Correction Capability } & $\begin{array}{l}\text { The Allowable } \\
\text { Strokes of the Rod }\end{array}$ \\
\hline $\begin{array}{c}\text { Translation along } \\
\text { the } x \text {-axis }\end{array}$ & $-92 \sim+92 \mathrm{~mm}$ & $\begin{array}{c}\text { Rotation around } \\
\text { the } x \text {-axis }\end{array}$ & $-18^{\circ} \sim+18^{\circ}$ & \multirow{3}{*}{$140 \sim 210 \mathrm{~mm}$} \\
\hline $\begin{array}{c}\text { Translation along } \\
\text { the } y \text {-axis }\end{array}$ & $-82 \sim+82 \mathrm{~mm}$ & $\begin{array}{l}\text { Rotation around } \\
\text { the } y \text {-axis }\end{array}$ & $-20^{\circ} \sim+20^{\circ}$ & \\
\hline $\begin{array}{c}\text { Translation along } \\
\text { the } z \text {-axis }\end{array}$ & $+122 \sim+196 \mathrm{~mm}$ & $\begin{array}{l}\text { Rotation around } \\
\text { the } z \text {-axis }\end{array}$ & $-34^{\circ} \sim+34^{\circ}$ & \\
\hline
\end{tabular}

It should be noted here that the soft tissue surrounding the bone will also be stretched to adapt to the growth of new bone tissue during distraction osteogenesis. The distraction osteogenesis is a very slow process ( $1 \mathrm{~mm} /$ day and four daily steps), which will reduce the damage of soft tissues and will not exceed the patient's tolerance of pain. Thus, the working ability of the external fixator is not restricted by the musculoskeletal system, which only causes a certain resistance to the operation of the external fixator during the correction.

\subsection{Trajectory Generating}

The parameters of the deformed bone and external fixator are designed as follows: the deformed bone has $8^{\circ}$ and $13^{\circ}$ angulation deformities around the $x$ and $y$-axes, respectively, $8 \mathrm{~mm}$ and $5 \mathrm{~mm}$ translation deformities along the $x$ and $y$-axes, respectively, $10^{\circ}$ rotation deformity around the $z$-axis, and $20 \mathrm{~mm}$ shortened deformity along the $z$-axis in the frame $O-x y z$. The diameter of both the proximal and distal ring is $160 \mathrm{~mm}$. For the above parameters of deformed bone, the translation deformity along the $z$-axis is the largest $(20 \mathrm{~mm})$. Therefore, it is assumed that the correction time is 20 days to ensure that the optimal correction rate is $1 \mathrm{~mm} /$ day along the $z$-axis. Meanwhile, the parameters can also ensure that the correction rate in the other five directions does not exceed $1 \mathrm{~mm} /$ day. The patient needs to wear the external fixator all the time and needs to maintain the fixator's stability. When the new bone tissue is completely calcified, the external fixator can be removed [18].

In reference to [6], it is assumed that the growth interface of bone is a circle centered on the central point of the bone end and that the new bone tissue fills the volume swept by the growth interface. A discrete set of target positions are fitted by MATLAB (R2011a, The Math Works, Inc., Novi, MI, USA) to generate the correction trajectory. The resulting bone shape, the volume swept by the growth interface, is then generated by fitting a series of growth interfaces. Due to the small magnitude of deformity parameters, a circle with a diameter of $8 \mathrm{~mm}$ is adopted to indicate the growth interface to visually and clearly express the characteristics of the new bone shape.

The correction trajectory and bone shape under the scheme of equal joint adjustment are generated through the numerical method, as shown in Figure 7. The generated trajectory is a smooth spatial curve, and the bone shape has a smooth curvature. The angulation deformities and translation deformities at the target position are completely corrected and the proximal and distal bone axes are aligned along the anatomical axis of the tibia (the axes axi1 and axi2 are aligned in the vertical direction). The advantage of this strategy is that it avoids the complicated process of calculating the correction prescription. There is a nonlinear mapping relationship between the joint and operating space of the frame, which causes the possibility that the adjustment value of the bone position may be too high or too small during the correction, and it also causes the orientation of the growth interface to change unevenly. 
(a)

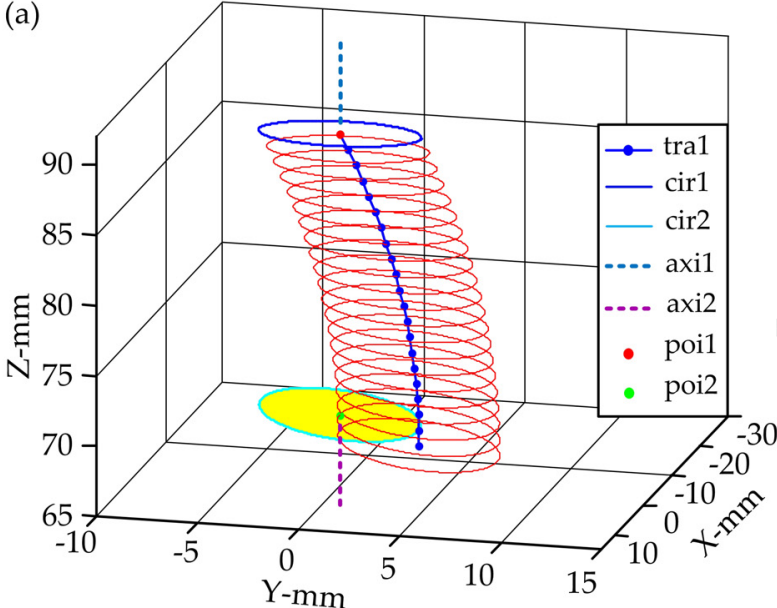

(b)

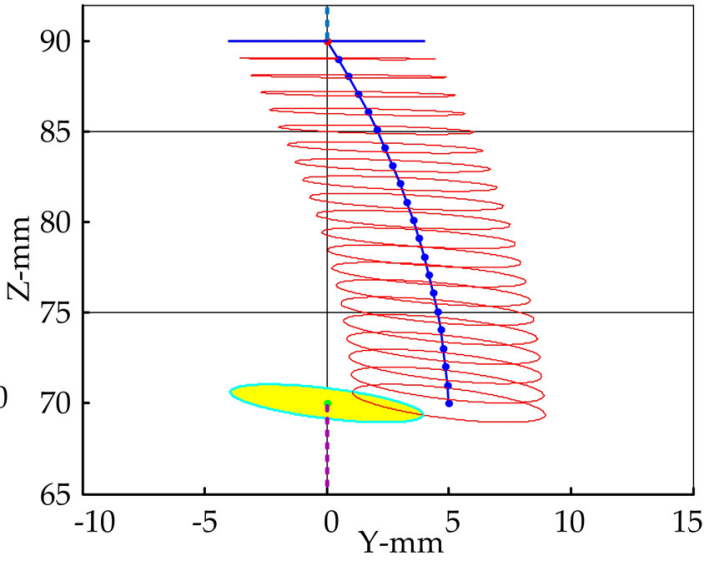

Figure 7. (a) The shape and trajectory of the bone with equal joint adjustment. (b) The view in the $\mathrm{Y}-\mathrm{Z}$ plane. The trajectory of tra1 represents the bone trajectory. The circles cir1 and cir2 denote the growth interface in the last step and the proximal bone end plane, respectively. The axes of axi1 and axi2 represent the axes of the distal and proximal bone segments, respectively.

The correction trajectory and bone shape of the given case are generated according to the strategy of joint adjustment for equal bone distraction, which can be found in Figure 8. The bone trajectory is a smooth and uniformly spaced linear trajectory and the shape of the new bone tissue is straight. The axis of distal bone is aligned with the anatomical axis at the desired position (the axes axi1 and axi2 are aligned in the vertical direction). The orientation of the bone end-plane changes uniformly, and there is no bone end interference between adjacent growth interfaces until the correction is completed. The strategy takes the shortest growth path and can plan the position and orientation of the bone segment simultaneously. The advantage of the strategy is that it avoids bone end-face interference, uneven traction, and shortening of the correction cycle, and finally, a smooth and uniformly spaced linear trajectory is obtained.

(a)

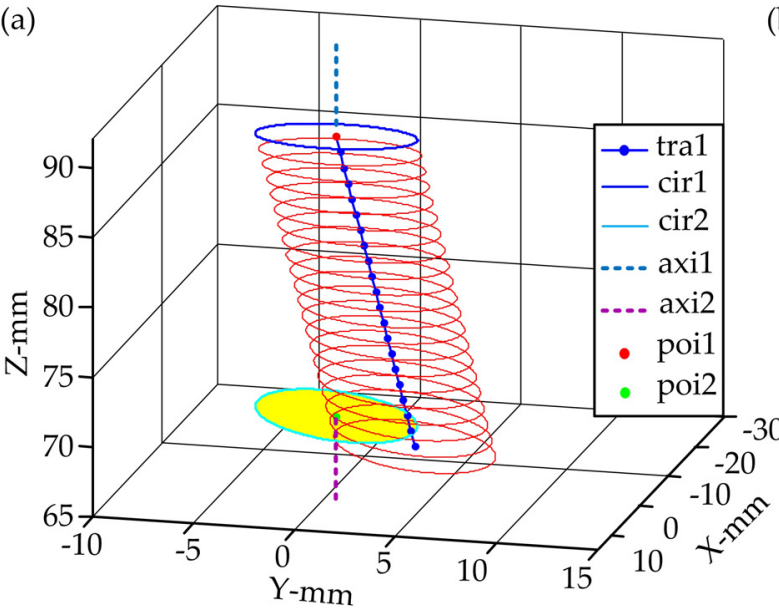

(b)

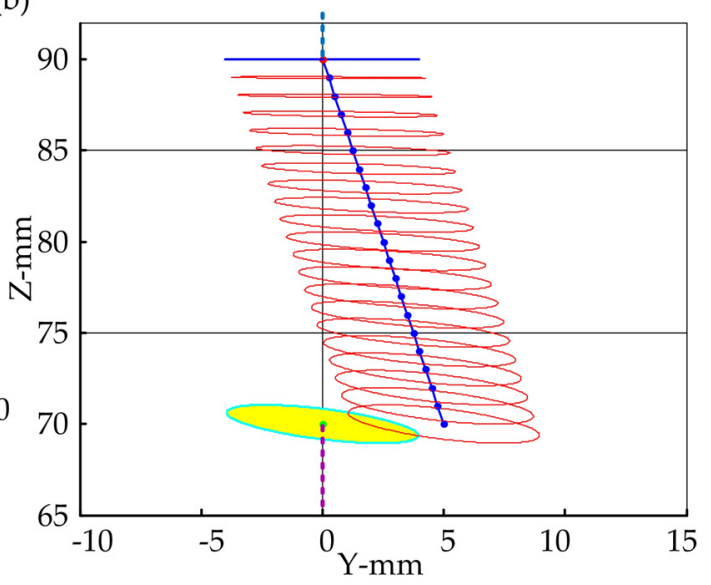

Figure 8. (a) The shape and trajectory of the bone with joint adjustment for equal bone distraction.

(b) The view in the $\mathrm{Y}-\mathrm{Z}$ plane.

\subsection{Trajectory Verification}

An experiment utilizing an artificial tibia model and the developed external fixator is designed to perform the two correction strategies. The artificial tibia model is osteotomized into two segments, and the proximal and distal bone segments are vertically and rigidly fixed to the proximal ring and 
the distal ring, respectively. The length of the proximal bone segment is $88 \mathrm{~mm}$, and the length of the distal bone segment is $73 \mathrm{~mm}$. The diameter of the proximal ring and the distal ring are both $220 \mathrm{~mm}$ and the distance of $A_{i} A_{j}(i j=16,23,45)$ is $32 \mathrm{~mm}$. The minimum and maximum allowable strokes of each of the telescopic rods are set to $140 \mathrm{~mm}$ and $210 \mathrm{~mm}$, respectively.

The preset correction trajectory based on two different correction strategies is demonstrated using the developed electromotor-driven parallel external fixator. In the initial position, the deformed tibia has a six-directional deformity. In the final position, the displacement deformity, angular deformity, shortening deformity, and rotation deformity are corrected, and the axis of the proximal and distal bone segments are aligned. The graphical stages of deformity correction with the sequential adjustment are shown in Figure 9.
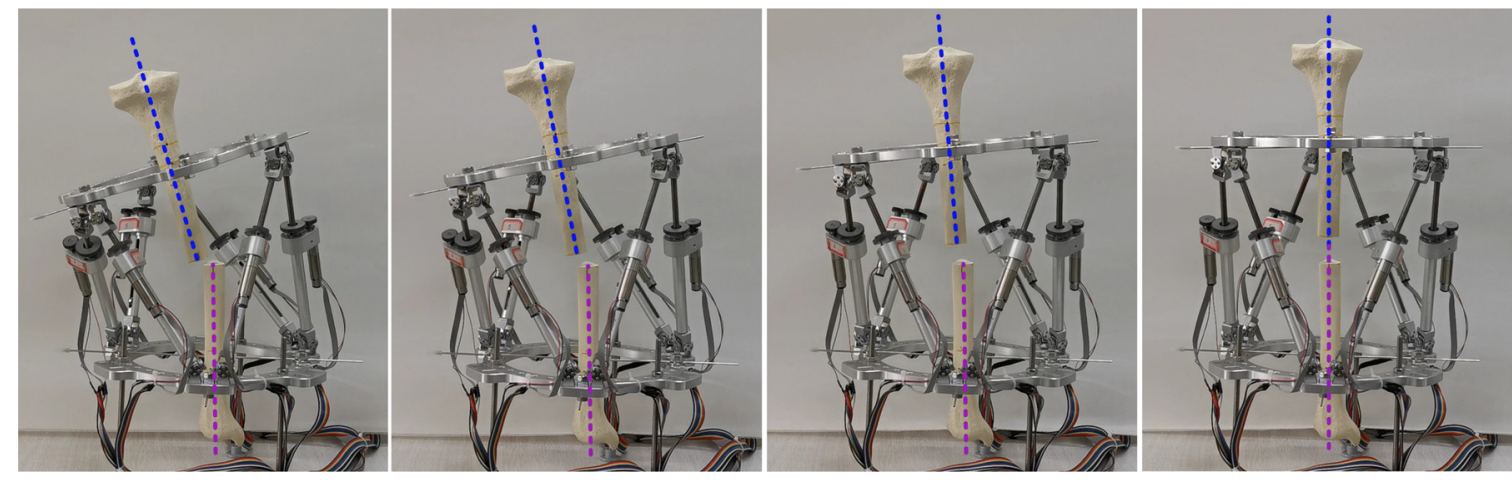

Figure 9. The graphical stages of deformity correction with sequential adjustment.

Meanwhile, the theoretical and actual values of each rod length during correction are acquired under two different correction strategies and the ranges of motion of each rod are as follows: $l_{1}, l_{5}, l_{6} \subset(167,195) \mathrm{mm}$ and $l_{2}, l_{3}, l_{4} \subset(169,201) \mathrm{mm}$, as shown in Figures 10 and 11 , which belongs to the reachable ROM of each telescopic rod. The length variations during the correction with equal joint adjustment show a linear trend. The initial rod lengths are different, and the changes in rods $l_{4}, l_{5}$, and $l_{6}$ are large. The external fixator is in a neutral position when it is corrected to the final position, and the final rod lengths tend to be the same, as shown in Figure 10. The changing trend of the length of each rod with the strategy of joint adjustment for equal bone distraction is similar to that of the strategy of equal joint adjustment; the difference is that the trend of length change with this strategy is not linear, as shown in Figure 11.

(a)

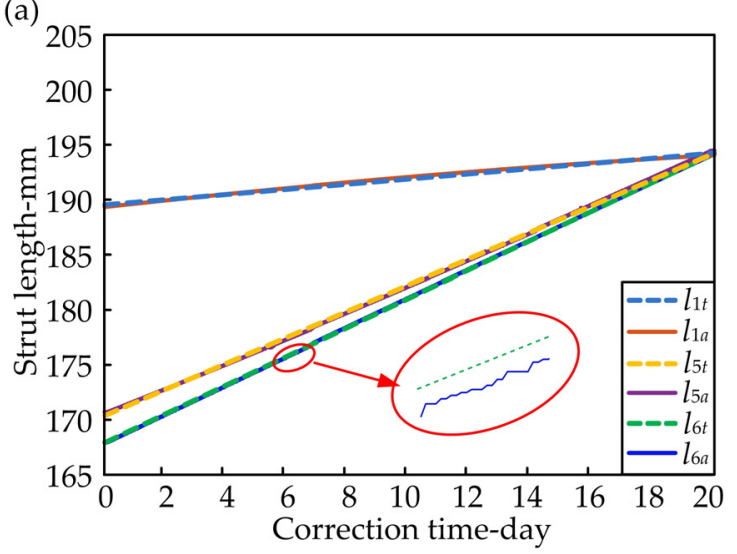

(b)

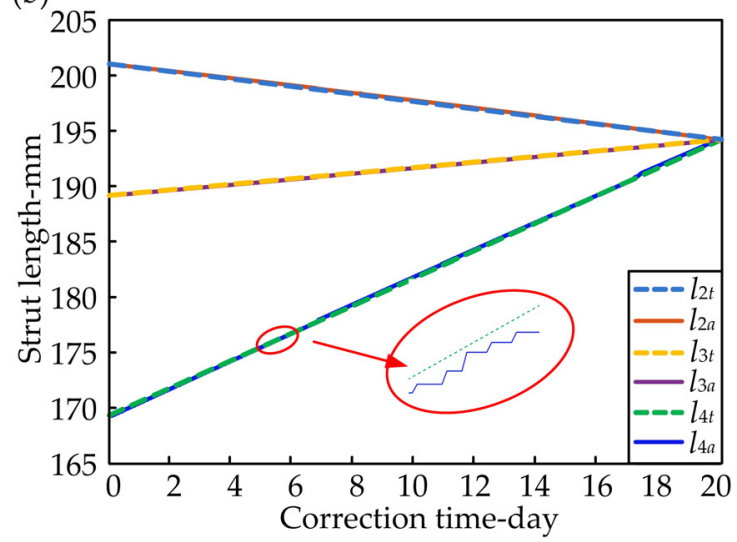

Figure 10. Theoretical $\left(l_{i t}\right)$ and actual $\left(l_{i a}\right)$ values of rod length during correction with equal joint adjustment. (a) Theoretical $\left(l_{i t}\right)$ and actual $\left(l_{i a}\right)$ values of length of rod $1, \operatorname{rod} 5$ and rod 6 . (b) Theoretical $\left(l_{i t}\right)$ and actual $\left(l_{i a}\right)$ values of length of $\operatorname{rod} 2, \operatorname{rod} 3$ and $\operatorname{rod} 4$. 
(a)

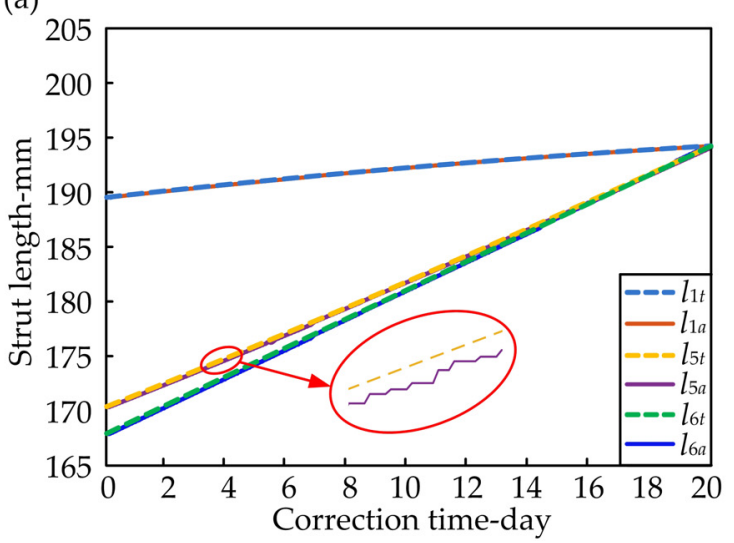

(b)

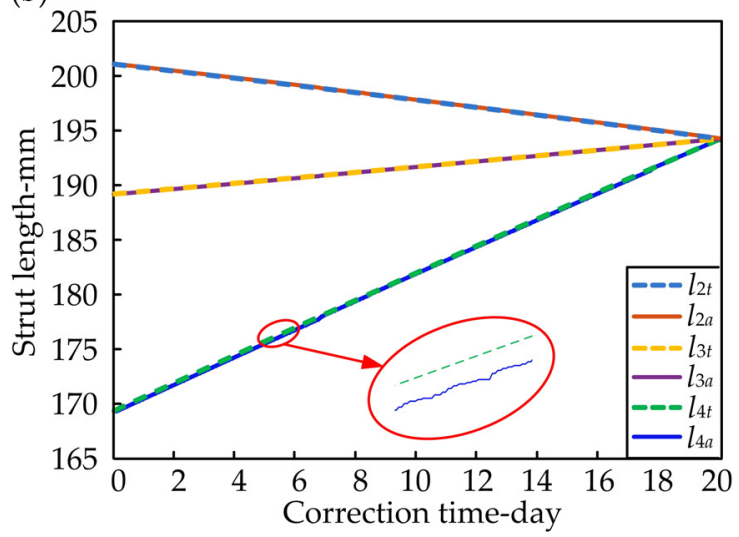

Figure 11. Theoretical $\left(l_{i t}\right)$ and actual $\left(l_{i a}\right)$ value of rod length during correction with joint adjustment for equal bone distraction. (a) Theoretical $\left(l_{i t}\right)$ and actual $\left(l_{i a}\right)$ values of length of rod 1 , rod 5 and $\operatorname{rod} 6$. (b) Theoretical $\left(l_{i t}\right)$ and actual $\left(l_{i a}\right)$ values of length of $\operatorname{rod} 2$, rod 3 and $\operatorname{rod} 4$.

The deviations between the actual values and the theoretical values of the rod length are also recorded during the correction with the two different strategies, as shown in Figure 12. The deviations of rods $l_{1}, l_{2}$ and $l_{3}$ are small, kept within $0.05 \mathrm{~mm}$, and the whole moving process is smooth with very small fluctuations. The deviations of rods $l_{4}, l_{5}$, and $l_{6}$ are maintained between $0.05 \mathrm{~mm}$ and $0.15 \mathrm{~mm}$, and the whole moving process is smooth with slight fluctuations.

(a)

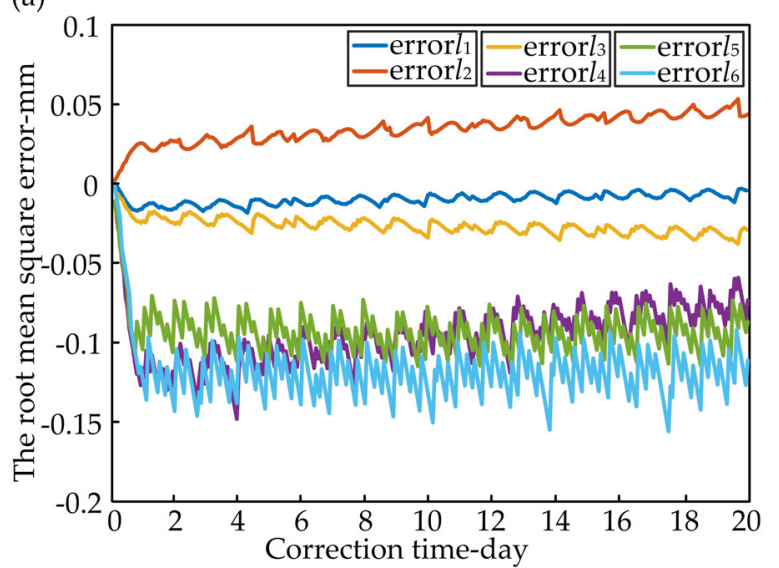

(b)

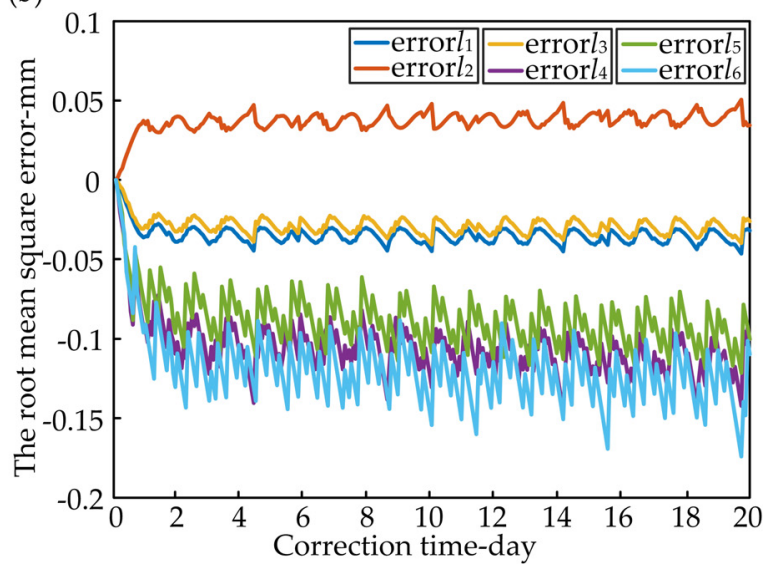

Figure 12. The deviations between the actual values and the theoretical value of the length of the rods. (a) The deviation with the strategy of equal joint adjustment. (b) The deviation with the strategy of joint adjustment for equal bone distraction.

The root mean square error of each rod is collected in Table 2, and their average root mean square error is $0.0712 \mathrm{~mm}$, which is sufficient to meet the accuracy requirements for deformity correction in orthopedic surgery. The results show that the developed electromotor-driven parallel external fixator meets the requirements of deformity correction with high correction accuracy and can accurately realize the preset correction trajectory to obtain better clinical correction results.

Table 2. The root mean square error of each rod.

\begin{tabular}{ccccccccc}
\hline & $\operatorname{Rod} l_{i}$ & $l_{1}$ & $l_{2}$ & $l_{3}$ & $l_{4}$ & $l_{5}$ & $l_{6}$ & Average Value \\
\hline Root mean & $\begin{array}{c}\text { Equal joint adjustment } \\
\text { square error }\end{array}$ & 0.0185 & 0.0306 & 0.0355 & 0.1046 & 0.1084 & 0.1275 & 0.0712 \\
& $\begin{array}{c}\text { Joint adjustment for equal } \\
\text { bone distraction }\end{array}$ & 0.0362 & 0.0384 & 0.0304 & 0.1088 & 0.0917 & 0.1242 & \\
\hline
\end{tabular}




\section{Discussion}

External fixators are widely used in orthopedics and allow for gradual correction, based on distraction osteogenesis (DO), for fracture reduction and deformity correction $[19,20]$. In terms of the configuration of the external fixator, the Ilizarov external fixator is the most mature ring fixator in clinical applications. It is a modular reconfigurable medical device in the mechanical sense. One of its main characteristics is that it can assemble a frame configuration that meets clinical needs through component assembly and configuration design according to different indications, which makes the Ilizarov frame have the advantages of flexible component assembly and diverse configuration designs. However, due to the complexity of the structure and the diversity of bone deformities, the Ilizarov frame technology requires a high level of clinical experience and medical skills, which makes the clinicians' learning curve for the use of the external fixator longer [5,21]. The external fixator is suitable for single-plane deformity correction, not multi-plane correction, and has achieved good clinical results, especially in limb lengthening [22,23]. The TSF (Taylor Spatial Frame) external fixator based on the Stewart platform can achieve spatial six-axis correction and can maintain continuous stability during the correction [5,8]. It has high transverse stiffness and accurately repeated positioning accuracy, which is conducive to bone growth. Besides, it has strong posture manipulation capabilities and a closed kinematic solution, which has been widely promoted and applied in the field of orthopedic deformity correction. The main advantage of TSF is that it has universal applicability to deformity and can be used for single-plane deformity correction as well as multi-plane complex deformity correction.

In addition to TSF, which is widely used in clinical applications and research, Ortho-SUV Frame (OSF) [24,25], PoliHex [12], TL-Hex [26], etc. are also well known. Traditionally, the rods' lengths are operated manually by the patient several times a day during the healing process. This involvement of the patient will ensure the patient's compliance and accumulative adjustment error. To reduce the patient's daily compliance with the external fixator caused by manual adjustment, $R$. Wendlandt et al. [12] developed an "intelligent" external fixator for fracture reduction, which allows movements of the bone fragments in all six degrees of freedom. Du et al. [27] reclassified fracture types using a stereolithography model and developed a teleoperation robot system based on visual servos to verify the effectiveness of the preoperative reduction path with fracture reduction experiments. M. Faschingbauer et al. [14] used an infrared tracking system to measure the positioning accuracy of a Hexapod ${ }^{\circledR}$ fixator and verified that the external fixator has high accuracy. Several researchers have described the advantages and precision of similar mechanisms [13-15]. A. M. Amaro et al. [28]. investigated the effect of external fixator configurations on the dynamic compression load through an experiment and numerical study. The accuracy of the external fixator during the implementation of the preset correction trajectory is also the main problem to be solved at present.

The developed electromotor driven parallel external fixator allows the deformity correction of any multi-planar deformity. In terms of mechanical structure, the lead screw is adopted with a single thread T thread ( $p=1 \mathrm{~mm}$ ), which has a good self-locking function. To increase the flexibility of the structure and avoid joint abrasion during correction, rolling bearings are used at screw nuts, Hooke hinges, and spherical joints. The control system adopts the distributed control mode of upper computer + lower computer, which makes it possible to automatically adjust the external fixator according to the correction strategy. The fixator system can provide a larger force/moment with $F_{\max }=2.52 \mathrm{KN}$ to balance the corrective resistance from muscles and soft tissues during the correction. The lower output speed with $0.89 \mathrm{~mm} / \mathrm{s}$ provided by the fixator system allows for very slow and continuous movement, which is effective for the safety of patients. Therefore, the external fixator system can not only achieve high deformity correction accuracy but also can be used as a teaching tool for clinicians to learn orthopedic deformity correction techniques in order to help them more intuitively and proficiently learn distraction osteogenesis (DO) and external fixation techniques. Meanwhile, combined with the development of computer-aided software, clinicians can perform preoperative simulation of the correction plan to ensure that the formulated correction strategy is safe and reliable, including frame configuration selection, preoperative trajectory simulation, preoperative limb-rod 
interference detection, and three-dimensional simulation of the correction process. Therefore, the external fixator system has high clinical application and teaching value.

During distraction osteogenesis, the soft tissue surrounding the bone will also be stretched to adapt to the growth of new bone tissue. The distraction osteogenesis is a very slow process $(1 \mathrm{~mm} /$ day and four daily steps), which will reduce the damage of soft tissues and will not exceed the patient's tolerance of pain. When the electromotor-driven fixator is used for correction, multiple rounds of adjustment per day are also followed, and the correction amount per day is $1 \mathrm{~mm}$ to avoid the unbearable pain caused by the excessive correction amount. The working capability of the external fixator is not restricted by the musculoskeletal and soft tissues, the main source of resistance to the operation of the external fixator, which only causes a certain resistance to the operation of the external fixator. It should be noted here that the control strategy is position control based on PID (Proportion Integration Differentiation), not force control. Theoretically, the output force of each strut is not a constant value and the output force depends on the resistance from the musculoskeletal and soft tissues during the correction [29]. As long as the resistance is less than the maximum output force that the motor can achieve, the resistance from the musculoskeletal can be balanced by the external fixator. As we all know, the parallel external fixator has the advantage of strong rigidity and can provide sufficient strength. Each strut of the developed parallel external fixator can provide a maximum output force of $2.52 \mathrm{KN}$, which is enough to offset the resistance of the soft tissue and musculoskeletal system during the correction.

In the future, a higher energy density battery and a more integrated control box will be used to reduce its volume and size, and the communication transmission between the PC and the control box will be changed from wired transmission to wireless transmission, which can allow the software to be used on more portable stand-alone devices (such as iPad) to reduce the difficulty of nursing during deformity correction. The force sensor will be installed at the end of the rod of the external fixator to monitor the force during the healing process. By studying the mechanism of dynamic correction force on the functional reconstruction of muscle and bone systems, dynamic correction trajectory planning based on quantitative monitoring and prediction of correction effect will also be carried out.

\section{Conclusions and Future Work}

In this work, an electromotor-driven parallel external fixator for deformity correction was developed-a hexapod external fixator based on the Steward platform. It can be automatically adjusted with the assistance of computer-aided software, according to the adjustment strategy, to reduce the involvement of the patient. The numerical searching method was utilized to investigate the ROM of the fixator, described as the size of the motion volume, to more intuitively assess the correction capability of the external fixator. Two deformity correction strategies based on different trajectory control methods were proposed, and the correction trajectories and bone shapes were generated through the numerical method to study their characteristics. The artificial bone model and the developed external fixator were used to verify the trajectory of the two proposed correction strategies. Experimental results show that the electromotor-driven parallel external fixator system can correct any three-dimensional deformity in a single plane or multiple planes and achieve a preset correction trajectory with high accuracy. The root mean square error between the theoretical and actual rod length of the frame is $0.0172 \mathrm{~mm}$. The developed external fixator can also be used as a teaching tool for medical training of clinicians in order to learn deformity correction technology.

Author Contributions: Formal analysis, G.L.; Funding acquisition, J.L. and M.D.; Methodology, G.L. and J.L.; Validation, G.L. and S.Z.; Writing-original draft, G.L.; Writing—review and editing, G.L., S.Z. and M.D. All authors have read and agreed to the published version of the manuscript.

Funding: This work was supported by the Beijing Natural Science Foundation under Grant No. 3171001, the General Program of Science and Technology Development Project of Beijing Municipal Education Commission under Grant No. KM202010005021, the Beijing Natural Science Foundation under Grant No. 3204036, the National Natural Science Foundation of China under Grants No.51675008 and No. 61903011, the National Key Research and Development Project under Grant No.2018YFB1307004, and the Beijing Postdoctoral Research Foundation under Grant No. Q6001002201901. 
Acknowledgments: The authors would like to acknowledge the National Research Center for Rehabilitation Technical Aids for its contribution to the implementation of correction strategies.

Conflicts of Interest: The authors declare no conflict of interest.

\section{References}

1. Sangkaew, C. Distraction osteogenesis for the treatment of post traumatic complications using a conventional external fixator: A novel technique. Injury 2005, 36, 185-193. [CrossRef]

2. Block, M.S.; Cervini, D.; Chang, A.; Gottsegenet, G.B. Distraction Osteogenesis. J. Pediatr. Orthopaed. 2015, 53, 561-565.

3. Birch, J.G.; Samchukov, M.L. Use of the Ilizarov Method to Correct Lower Limb Deformities in Children and Adolescents. J. Am. Acad. Orthop. Surg. 2004, 12, 144-154. [CrossRef] [PubMed]

4. Lan, X.; Zhang, L.; Peifu, T.; Xia, H.; Li, G.; Peng, A.; Han, Y.; Yuan, B.; Xu, W. S-osteotomy with lengthening and then nailing compared with traditional Ilizarov method. Int. Orthop. 2013, 37, 1995-2000. [CrossRef]

5. Dammerer, D.; Kirschbichler, K.; Donnan, L.; Kaufmann, G.; Krismer, M.; Biedermann, R. Clinical value of the Taylor Spatial Frame: A comparison with the Ilizarov and Orthofix fixators. J. Child. Orthop. 2011, 5, 343-349. [CrossRef]

6. Wu, Y.Y.; Plakseychuk, A.; Shimada, K. Computer-aided surgical planner for a new bone deformity correction device using axis-angle representation. Med. Eng. Phys. 2014, 36, 1536-1542. [CrossRef]

7. Mutlu, H.; Akçali, I.D.; Gulsen, M. A Mathematical Model for the Use of a Gough-Stewart Platform Mechanism as a Fixator. J. Eng. Math. 2005, 54, 119-143. [CrossRef]

8. Ganger, R.; Radler, C.; Speigner, B.; Grill, F. Correction of post-traumatic lower limb deformities using the Taylor spatial frame. Int. Orthop. 2009, 34, 723-730. [CrossRef]

9. Avşar, E.; Ün, K. Automatic 3D modeling and simulation of bone-fixator system in a novel graphical user interface. Inform. Med. Unlocked 2016, 2, 78-91. [CrossRef]

10. Du, H.; Hu, L.; Li, C.; He, C.; Zhang, L.; Tang, P. Preoperative trajectory planning for closed reduction of long-bone diaphyseal fracture using a computer-assisted reduction system. Int. J. Med. Robot. Comput. Assist. Surg. 2014, 11, 58-66. [CrossRef]

11. Du, H.; Hu, L.; Li, C.; Wang, T.; Zhao, L.; Li, Y.; Mao, Z.; Liu, D.; Zhang, L.; He, C.; et al. Advancing computer-assisted orthopaedic surgery using a hexapod device for closed diaphyseal fracture reduction. Int. J. Med. Robot. Comput. Assist. Surg. 2014, 11, 348-359. [CrossRef] [PubMed]

12. Seide, K.; Faschingbauer, M.; Wenzl, M.E.; Weinrich, N.; Juergens, C. A hexapod robot external fixator for computer assisted fracture reduction and deformity correction. Int. J. Med. Robot. Comput. Assist. Surg. 2004, 1, 64-69. [CrossRef] [PubMed]

13. Li, J.; Zhao, X.; Hu, X.; Tao, C.; Ji, R. Numerical investigation of the relationship between pin deviations and joint coordinates of a unilateral external fixator. Clin. Biomech. 2018, 53, 107-116. [CrossRef] [PubMed]

14. Faschingbauer, M.; Heuer, H.J.D.; Seide, K.; Wendlandt, R.; Münch, M.; Jürgens, C.; Kirchner, R. Accuracy of a hexapod parallel robot kinematics based external fixator. Int. J. Med. Robot. Comput. Assist. Surg. 2014, 11, 424-435. [CrossRef] [PubMed]

15. Li, C.; Wang, T.; Hu, L.; Zhang, L.; Du, H.; Wang, L.; Luan, S.; Tang, P. Accuracy Analysis of a Robot System for Closed Diaphyseal Fracture Reduction. Int. J. Adv. Robot. Syst. 2014, 11, 1-11. [CrossRef]

16. Zuo, S.; Dong, M.; Li, J.; Tao, C.; Ji, R. Configuration design and correction ability evaluation of a novel external fixator for foot and ankle deformity treated by U osteotomy. Med. Biol. Eng. Comput. 2020, 58, 541-558. [CrossRef]

17. Paley, D. History and Science Behind the Six-Axis Correction External Fixation Devices in Orthopaedic Surgery. Oper. Tech. Orthop. 2011, 21, 125-128. [CrossRef]

18. Vito, G.R.; Talarico, L.M.; Kanuck, D.M. Use of external fixation to correct deformities of the lower leg. Clin. Podiatr. Med. Surg. 2003, 20, 119-157. [CrossRef]

19. Nakase, T.; Kitano, M.; Kawai, H.; Ueda, T.; Higuchi, C.; Hamada, M.; Yoshikawa, H. Distraction osteogenesis for correction of three-dimensional deformities with shortening of lower limbs by Taylor Spatial Frame. Arch. Orthop. Trauma Surg. 2009, 129, 1197-1201. [CrossRef] 
20. Niculescu, B.; Faur, C.I.; Tataru, T.; Diaconu, B.M.; Cruceru, M. Investigation of Biomechanical Characteristics of Orthopedic Implants for Tibial Plateau Fractures by Means of Deep Learning and Support Vector Machine Classification. Appl. Sci. 2020, 10, 4697. [CrossRef]

21. Morsy, A.; Tsuchiya, H.; Matsubara, H.; Kabata, T.; Tomita, K. Ilizarov deformity correction of the lower limbs in Ellis-van Creveld syndrome. J. Orthop. Sci. 2007, 12, 505-509. [CrossRef] [PubMed]

22. Bor, N.; Rubin, G.; Rozen, N. Ilizarov Method for Gradual Deformity Correction. Oper. Tech. Orthop. 2011, 21, 104-112. [CrossRef]

23. Rozbruch, S.R.; Helfet, D.L.; Blyakher, A. Distraction of hypertrophic nonunion of tibia with deformity using Ilizarov/Taylor Spatial Frame-Report of two cases. Arch. Orthopaed. Trauma Surg. 2002, 122, 295-298. [CrossRef] [PubMed]

24. Takata, M.; Vilensky, V.A.; Tsuchiya, H.; Solomin, L.N. Foot Deformity Correction with Hexapod External Fixator, the Ortho-SUV Frame ${ }^{\mathrm{TM}}$. J. Foot Ankle Surg. 2013, 52, 324-330. [CrossRef]

25. Skomoroshko, P.V.; Vilensky, V.A.; Hammouda, A.I.; Fletcher, M.D.A.; Solomin, L.N. Mechanical rigidity of the Ortho-SUV frame compared to the Ilizarov frame in the correction of femoral deformity. Strat. Trauma Limb Reconstr. 2015, 10, 5-11. [CrossRef]

26. Riganti, S.; Nasto, L.A.; Mannino, S.; Marrè, B.G.; Boero, S. Correction of complex lower limb angular deformities with or without length discrepancy in children using the TL-Hex hexapod system: Comparison of clinical and radiograph- ical results. J. Pediatr. Orthopaed. B 2019, 28, 214-220. [CrossRef]

27. Li, C.; Wang, T.; Hu, L.; Zhang, L.; Du, H.; Zhao, L.; Wang, L.; Peifu, T. A visual servo-based teleoperation robot system for closed diaphyseal fracture reduction. Proc. Inst. Mech. Eng. Part H J. Eng. Med. 2015, 229, 629-637. [CrossRef]

28. Amaro, A.M.; Paulino, M.; Roseiro, L.; Neto, M.A. The Effect of External Fixator Configurations on the Dynamic Compression Load: An Experimental and Numerical Study. Appl. Sci. 2019, 10, 3. [CrossRef]

29. Dong, M.; Fan, W.; Li, J.; Zhou, X.; Rong, X.; Kong, Y.; Zhou, Y. A New Ankle Robotic System Enabling Whole-stage Compliance Rehabilitation Training. IEEE/ASME Trans. Mechatron. 2020, 1. [CrossRef]

Publisher's Note: MDPI stays neutral with regard to jurisdictional claims in published maps and institutional affiliations.

(C) 2020 by the authors. Licensee MDPI, Basel, Switzerland. This article is an open access article distributed under the terms and conditions of the Creative Commons Attribution (CC BY) license (http://creativecommons.org/licenses/by/4.0/). 